\title{
SPATIO-TEMPORAL ANALYSIS TO PREDICT ENVIRONMENTAL INFLUENCE ON MALARIA
}

\author{
Shahbaz Baig ${ }^{1}$, Muhammad Shahzad Sarfraz ${ }^{2}$ \\ ${ }^{1}$ Department of Computer Science, University of Gujrat, Gujrat Pakistan - 15051719-006@uog.edu.pk \\ ${ }^{2}$ Department of Computer Science, University of Gujrat, Gujrat Pakistan - $\underline{\text { shahzad.sarfraz@uog.edu.pk }}$
}

\section{Commission III, ICWG III/IVc}

KEY WORDS: Malaria, Prediction, Moran's I, Spatial Analysis and Environmental Influence

\section{ABSTRACT:}

Malaria is a vector borne disease which is a major cause of morbidity and mortality. It is one of the major diseases in the category of infectious diseases. The survival and bionomics of malaria is affected by environmental factors such as climatic, demographic and land-use/land-cover etc. Currently, a very few under developing countries are using Geo-informatics approaches to control this disease. Gujrat a district of Pakistan, is still under threat of malaria disease. Current research is carried on malaria incidents obtained from District Executive Officer of Health Gujrat. The objective of this study was to explore the spatio-temporal patterns of malaria in district Gujrat and to identify the areas being affected by Malaria. Furthermore, it has been also analyzed the relationship between malaria incident and environmental factors in highly favorable zones. Data is analyzed based on spatial and temporal patterns using (Moran's I). Moreover cluster and hot spots analysis were performed on the incident data. This study shows positive correlation with rainfall, vegetation index, population density and water bodies; while it shows positive and negative correlation with temperature in different seasons. However, variation between amount of vegetation and water bodies were observed. Finding of this research can help the decision makers to take preventive measures and reduce the morbidity and mortality related with malaria in Gujrat, Pakistan.

\section{INTRODUCTION}

Malaria is a vector borne disease which is a major cause of morbidity and mortality. More than two hundred million people are affected in 91 countries with an increase of 5 million cases over 2015 by malaria in a calendar year (WHO, 2017). It is one of the major diseases in the category of infectious diseases. Malaria is a major disease affected by environmental factors. The survival and bionomics of malaria is affected by climatic variables like rain fall, humidity, temperature etc. Currently, very few countries are using Geoinformation technology to control this disease (Coleman et al., 2007; Coleman et al., 2006). Geo-informatics is used worldwide to monitor malaria transmission attributes and its social and cultural aspects (Daash et al., 2009). There is a need to identify the hot spots where concentration of malaria vector borne disease is high based on climatic factors.

Around 3-5 billion cases of malaria are reported each year from which almost two million patients have lost their lives. Moreover, almost $80 \%$ of the cases of malaria are reported from Sub Saharan Africa (Abdulhakim, 2013). Mapping of malaria-risk zones in many parts of the world has been successfully validated by the integrated use of Geographical Information Systems (GIS) and Remote ensing (RS) (Sithiprasasnaet al., 2005; Donguset al., 2007). More than three hundred million people are at risk of malarial infection around the world as environmental factors are contributing in the transmission of disease and its seasonal patterns.

Many factors such as water bodies, rainfall, population density, temperature etc. are associated with malaria and its control (Palaniyandi, 2012). The use of GIS in mapping of malaria incidences is currently being used at regional, national, and international level and its impact is also increasing in prediction and control of malaria (Saxena et al., 2009). Spatio-temporal mapping of malaria disease can help to identify the areas of high risk which should be monitored and to develop strategies to control this disease (Zacarias et al., 2011). Maps generated by GIS software provide visual representation of those areas which are at high risk and need to be treated well to control malaria (Riedelet al., 2010). The use of geographical modeling of malaria distribution is a key to understand the spatial and temporal patterns. These patterns reflect the factors that causes malaria; diversity in the distribution of vectors and their contact with humans (Zacarias et al., 2010).

Use of GIS and spatial statistical methods are contributing in developing risk maps of malaria based on its distribution at regional and country level (Bhunia et al., 2010). Many studies used different approaches to model the spatial distribution of malaria due to their flexibility, robustness in mapping of disease and making decisions. To estimate the risk of malaria; statistical analysis are being performed in many countries around the world (Zacarias et al., 2010; Stensgaard et al., 2011).

To develop more effective and viable strategies to control malaria and its removal, there is a need of better understanding of spatio-temporal distribution of malaria vectors (Ferguson et al., 2010; Sinka et al., 2012). Therefore, main focus of this study is on identifying the hot spots of malarial cases based on several factors like rainfall, water bodies, population density etc. 


\section{MATERIALS AND METHODS}

\subsection{Study Area}

Gujrat, the $18^{\text {th }}$ largest city of Pakistan in terms of population, has been selected for this study. It is situated between two rivers i.e. Chenab and Jhelum. This area has been selected for this study because there are more possibilities of infectious diseases in this district due to its geographic location and environmental factors.

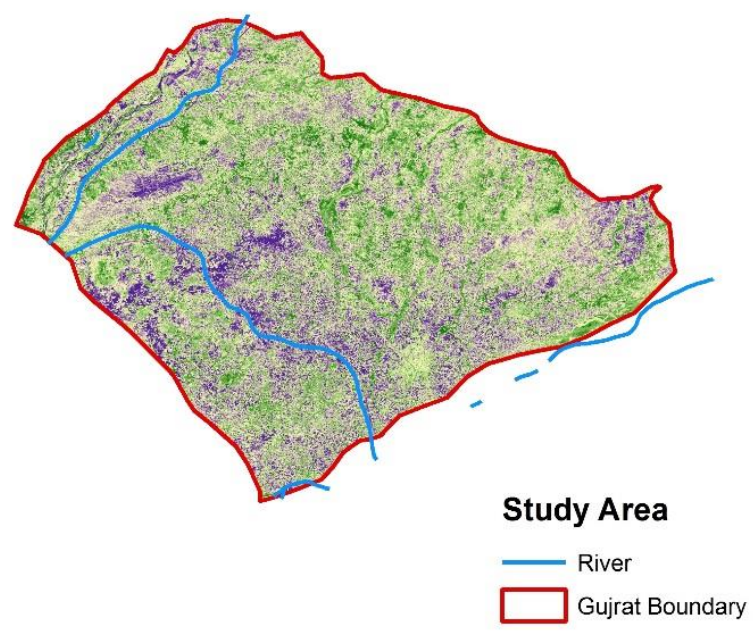

Figure 1. Study Area which represent boundary and river of Gujrat, Pakistan

\subsection{Data Collection}

Data of malaria cases was collected from District Health Office Gujrat. Temperature and rainfall data was extracted from satellite images and metrological stations data was obtained from Pakistan Meteorological Department (PMD). Landsat data was downloaded from earth explore website and Normalized Difference Vegetation Index (NDVI) and Normalized Difference Water Index (NDWI) was calculated using ArcMap software. Population density and water bodies have also been considered to carry on this study.

\subsection{Methodology}

Data gathered from District Executive Health Office Gujrat was analyzed statistically by considering gender and age of individuals. NDVI and NDWI were calculated using Landsat 8 data with the help of ArcMap and region of Gujrat is extracted for this study. Temperature and rainfall data downloaded through satellites and collected from PMD was analyzed to find its impact on malaria transmission. Regression analysis was performed to evaluate the relationship of malaria cases on the factors i.e. population density, NDVI, NDWI, rainfall and temperature. Further on, Moran's I tool is used to calculate spatial autocorrelation. It evaluates whether the pattern is clustered, dispersed or random (Oliveira et al., 2013). Hot spots of malaria were identified by considering all above parameters and relation of these parameters with disease occurrence and transmission.

\subsection{General Analysis}

\section{RESULTS}

General analysis shows that $54 \%$ males and $46 \%$ females were affected by malaria. Among all the cases $46 \%$ cases are of the children whose age is less than 10 years. While $22 \%$ cases are of age between 11-20 years, $19 \%$ are of between 21 40 years, $11 \%$ between $41-60$ years, and $2 \%$ cases are of age greater than 60 years. This shows that $68 \%$ of reported cases are of age 20 or less.

\begin{tabular}{|c|c|c|}
\hline Gender & Male & Female \\
\hline Cases \% & 54 & 46 \\
\hline
\end{tabular}

Table 1(a). General analysis of Malaria cases by Gender

\begin{tabular}{|l|l|l|l|l|l|}
\hline Age & $<=10$ & $11-20$ & $21-40$ & $41-60$ & $>60$ \\
\hline Cases \% & 46 & 22 & 19 & 11 & 2 \\
\hline
\end{tabular}

\subsection{Spatio Temporal Analysis}

\subsubsection{Rainfall and Malaria}

This study shows positive relation with rainfall. There was high amount of rain in months of July and August that grounds more places for mosquito breeding and it results in more malaria cases.

\subsubsection{Temperature and Malaria}

Temperature plays a vital role in spreading of vector borne diseases. Temperature between $20-35^{\circ} \mathrm{C}$ remained suitable for mosquito breeding and it shows both positive and negative correlation with temperature.

\subsubsection{Vegetation and Malaria}

Results show positive and negative correlation with malaria disease. It indicates that more vegetation are more suitable breeding sites for mosquitoes and it may results in more malaria transmission and occurrence.

\subsubsection{Other Factors and Malaria}

Water bodies and population density shows a variation in results. It has been observed that some areas having high population but resulted less malaria cases and vice versa. Similarly, water bodies such as ponds, rivers and streams showed variation in terms of malaria incidence.

\subsubsection{Moran' I and Hot Spots}

Overlay analysis is performed on all layers of data and Moran's I was calculated with Moran's index 0.294846 and zscore 62.420632. It shows that pattern is clustered. Hot spots using Getis-OrdGi* were identified by considering all the factors and risk map of Gujrat was prepared for malaria identification and elimination. 


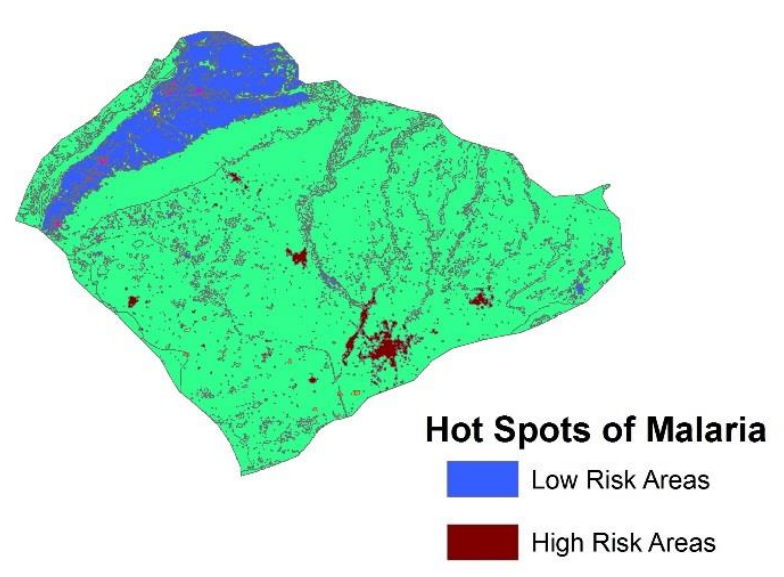

Figure 2. Dark red areas shows the hotspots for malaria based on given factors on risk map

\section{CONCLUSION}

This study was conducted to measure the effect of environmental factors such as rainfall, temperature, NDVI etc. on malaria. It shows positive relation with rainfall while there was no significant correlation found with the other factors. It is important for researchers to consider additional factors effects on malaria transmission such as socio-economic etc. to identify high risk areas for vector borne diseases. Such types of studies shall help policy and decision makers to take preventive measures to control and eliminate such types of diseases. Moreover, there is a dire need to investigate the relationship among different types of vector borne diseases which are causing heavy burden on low incoming countries.

\section{REFERENCES}

A. Daash, A. Srivastava, B. N. Nagpal, R. Saxena, and S. K. Gupta, 2009. Geographical information system (GIS) in decision support to control malaria - a case study of Koraput district in Orissa, India, Journal of Vector Borne Diseases, vol. 46, no.1, pp. 72-74.

Abdulhakim, A.2013. GIS and remote sensing integrated for malaria risk mapping, Kersa, Ethiopia, MSc. Thesis. Teri University, New Delhi.

Bhunia G, Kumar V, Kumar A, Das P, Kesari S.2010. The use of remote sensing in the identification of the ecoenvironmental factors associated with the risk of human visceral leishmaniasis (kala-azar) on the Gangetic plain, in north-eastern India. Ann Trop Med Parasitol 104: 35-53.

Dongus, S., D. Nyika, K. Kannady, D. Mtasiwa, H. Mshinda and U. Fillinger, 2007.Participatory mapping of target areas to enable operational larval source management to suppress malaria vector mosquitoes in Dar es Salaam, Tanzania. International Journal of Health Geographics 6:37. doi:10.1186/1476-072X-6- 37.

Ferguson HM, Dornhaus A, Beeche A, Borgemeister C, Gottlieb M, Mulla MS, Gimnig JE, Fish D, Killeen.2010 GF: Ecology: a prerequisite for malaria elimination and eradication. PLoS Med.
M. Coleman and J. Hemingway, 2007. Insecticide resistance monitoring and evaluation in disease transmitting mosquitoes, Journal of Pesticide Science, vol. 32, no. 2, pp. 69-76.

M. Coleman, B. Sharp, I. Seocharan, and J. Hemingway, 2006. Developing an evidence-based decision support system for rational insecticide choice in the control of African malaria vectors, Journal of Medical Entomology, vol. 43, no. 4, pp. 663-668.

Oliveira, E.C., E.S. Santos, P. Zeilhofer, R. SouzaSantos and M. Atanaka-Santos.2013. Geographic information systems and logistic regression for highresolution malaria risk mapping in a rural settlement of the southern Brazilian Amazon. Malaria Journal, doi:10.1186/1475-2875-12-420.

Palaniyandi, M.2012. The role of remote sensing and GIS for spatial prediction of vector-borne diseases transmission. Journal of Vector Borne Disease 49: 197-204.

Riedel N, Vounatsou P, Miller J, Gosoniu L, ChizemaKawesha E, Mukonka V \&Steketee R.2010. Geographical patterns and predictors of malaria risk in Zambia: Bayesian geostatistical modelling of the 2006 Zambia national malaria indicator survey (ZMIS) Malaria Journal, 9, 37.

Saxena, R., B.N. Nagpal, A. Srivastava, S.K. Gupta and A.P.2009. Application of spatial technology in malaria research \& control: Some new insights. Indian Journal of Medical Research 130:125-132.

Sinka ME, Bangs MJ, Manguin S, Rubio-Palis Y, Chareonviriyaphap T, Coetzee M, Mbogo CM, Hemingway J, Patil AP, Temperley WH, Gething PW, Kabaria CW, Burkot TR, Harbach RE, Hay SI:2012. A global map of dominant malaria vectors. Parasit Vectors.

Sithiprasasna, R., W.J. Lee, D.M. Ugsang and K.J.2005. Linthicum. Identification and characterization of larval and adult anopheline mosquito habitats in the Republic of Korea: Potential use of remotely sensed data to estimate mosquito distributions. International Journal of Health Geographics 4:1-11.

Stensgaard A, Vounatsou P, Onapa A, Simonsen P, Pedersen E, Rahbek C \&Kristensen T.2011. Bayesian geostatistical modelling of malaria and lymphatic filariasis infections in Uganda: predictors of risk and geographical patterns of coendemicity. Malaria Journal,10, 29.

WHO, 2017. "Roll back malaria: action or rhetoric? Round table discussion," Bulletin of the World Health Organization, vol.78, pp. 1450-1455.

Zacarias O \&Andersson M.2010. Mapping malaria incidence distribution that accounts for environmental factors in Maputo Province--Mozambique. Malaria Journal, 9, 79.

Zacarias Op \&Andersson M.2011. Spatial and temporal patterns of malaria incidence in Mozambique. Malaria Journal,10, 189. 